\title{
Solid state NMR characterization of phenylphosphonic acid encapsulated in SBA-15 and aminopropyl-modified SBA-15
}

\author{
Daniela Aiello ${ }^{\mathrm{a} b}$, Nicolas Folliet ${ }^{\mathrm{a}}$, Guillaume Laurent ${ }^{\mathrm{a}}$, Flaviano Testa ${ }^{\mathrm{b}}$, Christel Gervais ${ }^{\mathrm{a}}$, \\ Florence Babonneau ${ }^{\mathrm{a}}$, Thierry Azaïs ${ }^{\mathrm{a}, *}$ \\ a UPMC Univ Paris 06 \& CNRS, UMR 7574, Chimie de la Matière Condensée de Paris, Collège de France, 11, Place Marcelin Berthelot, F-75005 Paris, France \\ ${ }^{\mathrm{b}}$ Department of Chemical Engineering and Materials, CR-INSTM, University of Calabria, 87036 Arcavacata di Rende (CS), Italy
}

Keywords:

Mesoporous materials

Silica

Encapsulation

Phosphonic acid

Solid state NMR

\begin{abstract}
A B S T R A C T
We present in this communication that phenyl phosphonic acid can be efficiently loaded in mesoporous SBA-15 and aminopropyl-modified SBA powdered samples through the incipient wetness impregnation method. High amount of phosphonic acid can be reach up to $380 \mathrm{mg} / \mathrm{g}$ of sample. We use multinuclear solid state NMR as a method of choice for the indeep characterization of the samples. Thus we demonstrate that phosphonic acid molecules do not crystallize inside the pores. The molecules are highly mobile in SBA-15 because they are submitted to a confinement effect due to the mesoscopic size of the pores and consequently they exhibit a weak interaction with the silica walls. In the case of aminopropyl-modified SBA material, we show that the molecules are rigid and that they are in strong interaction with the aminopropyl groups. Moreover, a 2D double quantum ${ }^{1} \mathrm{H}$ NMR experiment recorded at high field and high spinning speed permit to propose a model of the phosphonate-aminopropyl interaction. The increase in spectral resolution due to the combination of high magnetic field and fast MAS rate allows also the assignment of ${ }^{1} \mathrm{H}$ resonances in aminopropyl-modified SBA matrix and notably allows the assignment of the protons resonance of the amino group.
\end{abstract}

\section{Introduction}

Since 2001 [1], there is a growing interest of the researchers for drug release systems based on ordered mesoporous silica such as MCM-41 and SBA-15. These materials possess the particularity to increasing the bioavailability of hydrophobic drugs and therefore are highly attractive for biomedical applications [2]. It was shown that the in vitro release kinetics of a given drug can be modulated by (i) the pore size [3], (ii) the topology of the mesoporous network [4], (iii) the chemical nature of the pore surface that controls the interactions between the silica walls and the encapsulated molecules [5]. Nowadays, based on the same concept, researchers develop mesoporous silica nanoparticles as cargos to deliver drugs directly inside the cells [6]. The fine characterization of the active molecule inside the vector material is crucial on a pharmaceutical point of view. Indeed, the nature of its physical state or its crystalline polymorph influences directly the dosage of the galenic form. Thus, solid state NMR is widely used for such a characterization [7] because of its ability to act as a local probe allowing the investigation of chemical environment around a given nucleus [8]. In particular, it was shown that ibuprofen (a common anti-inflammatory

\footnotetext{
* Corresponding author. Tel.: +33 (0) 1442715 43; fax: +33 (0) 144271504 E-mail address: thierry.azais@upmc.fr (T. Azaïs).
}

drug) encapsulated in MCM-41 materials exhibits an original physical state in-between a liquid and a solid whereas the bulk substance is crystalline at room temperature [9]. Indeed, the trapped molecules undergo fast dynamics due to the presence of a confinement effect [10]. Such effect which is still not fully understood implies a depression of the thermodynamical parameters such as phase transition temperatures and are observed for molecules confined in mesopores (diameter ranging from 2 to $50 \mathrm{~nm}$ ) in absence of strong interaction with the host matrix.

In this communication, we present the synthesis and the study of hybrid materials where phenylphosphonic acid (ФP) is encapsulated in SBA-15 and aminopropyl-modified SBA-15 materials through incipient wetness impregnation. SBA-15 are a class of siliceous mesoporous material discovered in 1998 characterized by an ordered 2D-hexagonal architecture and a narrow pore size distribution around 50-60 $\AA$ [11]. We use $\Phi P$ as a model molecule for bisphophonates (zoledronate, alendronate...) which are a class of molecules widely used as drug fighting osteoporosis and therefore helping bone regeneration. Indeed, it has been shown that alendronate can be efficiently encapsulated in mesoporous silica (MCM41 and SBA-15) grafted or not with aminopropyl groups [12]. It has been shown that the loading efficiency and the release kinetic are highly influenced by the nature of the chemical groups grafted onto the surface (aminopropyl, alkyl...) [13,14]. In this paper, we 
report the characterization of ФP confined in SBA-15 and aminopropyl-modified SBA-15 materials by various techniques including multinuclear solid-state NMR in order to get information on (i) the physical state of the confined phosphonate molecules and (ii) the nature of the phosphonate-silica interactions. Indeed, these informations are often neglected in previous reports although they are of primary importance in order to understand the in vitro release properties of the system and the consequent drug bioavailability. We demonstrate that it is possible to obtain these precious informations through ${ }^{1} \mathrm{H},{ }^{13} \mathrm{C}$ and ${ }^{31} \mathrm{P}$ solid state NMR. In particular, we studied the dynamical properties of the guest molecule through ${ }^{1} \mathrm{H}-{ }^{31} \mathrm{P}$ cross polarization $(\mathrm{CP})$ experiments at variable contact time and ${ }^{31} \mathrm{P}$ chemical shift anisotropy (CSA) measurements. The phosphonate-silica interface was investigated by two dimensional double quantum (DQ) ${ }^{1} \mathrm{H}$ experiments. The experiments were carried out at high field (16.4 Tesla) and very fast spinning speed (up to $65 \mathrm{kHz}$ ) in order to increase the ${ }^{1} \mathrm{H}$ spectral resolution which is usually poor compared to solution state NMR due to strong ${ }^{1} \mathrm{H}-{ }^{1} \mathrm{H}$ homonuclear dipolar couplings. Thus, we demonstrate that ФP molecules encapsulated in unmodified SBA-15 are submitted to confinment effects that increase their dynamical properties at room temperature compared to bulk ФP. On the other hand, ФP confined in aminopropyl-modified SBA-15 exhibit a rigid behavior due to the strong interaction of the molecules with the aminopropyl groups. Spatial proximities $(<5-6 \AA$ ) are elucidated that allow the proposition of a model for the phosphonate-aminopropyl interaction.

\section{Experimental}

\subsection{SBA-15 material synthesis}

The preparation of the SBA15 samples was similar to the method described by Zhao et al. [11]. The template solution was prepared by dissolving $4.0 \mathrm{~g}$ of Pluronic P123 (Fluka) in $104 \mathrm{~mL}$ of deionized water and $20 \mathrm{~mL}$ of $37 \mathrm{wt} . \% \mathrm{HCl}$ under stirring at $35^{\circ} \mathrm{C}$. Then $9.16 \mathrm{~mL}$ of tetraethylorthosilicate (TEOS) were added into the solution that was kept under magnetic stirring for $12 \mathrm{~h}$ at room temperature. The molar composition of final mixture was TEOS/ $\mathrm{HCl} / \mathrm{P} 123 / \mathrm{H}_{2} \mathrm{O}=1: 6.03: 0.017: 145$. The white precipitate was aged at $100{ }^{\circ} \mathrm{C}$ for $24 \mathrm{~h}$, then filtered, washed with deionised water and dried at $60^{\circ} \mathrm{C}$ for $12 \mathrm{~h}$. Calcination was carried out at $550{ }^{\circ} \mathrm{C}$ in $8 \mathrm{~h}$ in air. The free template sample is denoted as SBA in the forthcoming text.

\subsection{Aminopropyl-modified SBA material synthesis}

The process was carried out through post-grafting synthesis by reacting $1 \mathrm{~g}$ of the mesoporous materials previously activated in an oven at $120^{\circ} \mathrm{C}$ for $2 \mathrm{~h}$ with $1.33 \mathrm{~mL}$ of (3-aminopropyl)triethoxysilane (APTES - $\left(\mathrm{C}_{2} \mathrm{H}_{5} \mathrm{O}\right)_{3} \mathrm{Si}\left(\mathrm{CH}_{3}\right)_{3} \mathrm{NH}_{2}$; Aldrich) in $20 \mathrm{~mL}$ of toluene (Fluka) under magnetic stirring. The mixture was heated under reflux at $110^{\circ} \mathrm{C}$ overnight and the product was filtered, washed with toluene and dried at $100{ }^{\circ} \mathrm{C}$ overnight. The resulting amount of aminopropyl groups is $2.5 \mathrm{mmol}$ per gram of solid from thermogravimetric measurements. Amine-functionalized mesoporous silica material is denoted as SBA-NH in the forthcoming text.

\subsection{Phosphonic acid encapsulation}

The SBA and SBA-15-NH samples were activated at $100{ }^{\circ} \mathrm{C}$ for $12 \mathrm{~h}$. The encapsulation process was performed through incipient wetness procedure [15] by wetting $0.500 \mathrm{~g}$ of mesoporous samples with a solution of phenylphosphonic acid ( $\Phi \mathrm{P}-\mathrm{C}_{6} \mathrm{H}_{7} \mathrm{O}_{3} \mathrm{P}$; Fluka; $\left.0.012 \mathrm{~g} \mathrm{~cm}^{-3}\right)$ in ethanol. Then, the solvent was removed by heating at $70{ }^{\circ} \mathrm{C}$ overnight. This procedure was repeated three successive times. After the forth impregnation, the samples were quickly washed with $4 \mathrm{ml}$ of ethanol, under vacuum, in order to remove the excess of recrystallized $\Phi P$ and dried in an oven at $70^{\circ} \mathrm{C}$ overnight. The ФP-loaded samples are denoted as SBA-ФP and SBA-NH-ФP in the forthcoming text.

\subsection{Sample characterization}

Nitrogen adsorption/desorption isotherms were recorded at $77 \mathrm{~K}$ with a Micromeritics ASAP 2000 apparatus, after activation of the sample under vacuum $\left(1 \times 10^{-3} \mathrm{Torr}\right)$ at $350{ }^{\circ} \mathrm{C}$ for SBA or at $115^{\circ} \mathrm{C}$ for $6 \mathrm{~h}$ for SBA-ФP. The specific surface area $\mathrm{S}_{\mathrm{BET}}$ is calculated according to the standard BET method, [16] while the mean pore diameter is estimated by the $\mathrm{BJH}$ method using the desorption branch [17].

XRD patterns were recorded with a Philips PW 1830 diffractometer equipped with a $\mathrm{Cu}-\mathrm{K} \alpha$ source. Small angle measurements were performed in a $2 \theta$ range from 1 to $6^{\circ}$ with a step size of $0.002^{\circ}$ and a step time of $3 \mathrm{~s}$.

Solid state NMR experiments were performed on a Avance 300 Bruker spectrometer (7.0 Tesla) equipped with a double channel probe. Samples were packed in $4 \mathrm{~mm}$ zirconia rotors and spun at $5-14 \mathrm{kHz} .{ }^{1} \mathrm{H},{ }^{31} \mathrm{P},{ }^{29} \mathrm{Si}$ single pulse and ${ }^{1} \mathrm{H}-{ }^{31} \mathrm{P}$ and ${ }^{1} \mathrm{H}-{ }^{13} \mathrm{C}$ crosspolarization (CP) experiments were recorded with high power proton decoupling (spinal-64) during acquisition. Recycle delays (RD) for ${ }^{1} \mathrm{H}$ and ${ }^{31} \mathrm{P}$ NMR experiments were set to $2-10 \mathrm{~s}$, and $60 \mathrm{~s}$ for ${ }^{29} \mathrm{Si}\left(30^{\circ}\right.$ pulse), in order to get full relaxation of the magnetization before each scan. The EXSY (Exchange spectroscopy) experiments where recorded with a standard three pulses sequences where the evolution and the mixing delays (200 ms) where rotor-synchronized in order to avoid the reintroduction of unwanted interactions High field and high spinning speed solid state NMR experiments were performed on a Avance 700 Bruker spectrometer (16.4 Tesla) equipped with a double channel probe. Samples were packed in $1.3 \mathrm{~mm}$ zirconia rotors and spun at 60-67 kHz. Typically, ${ }^{1} \mathrm{H}$ single pulse and ${ }^{1} \mathrm{H}$ double quantum experiments (BABA sequence) were recorded with $v_{R F}\left({ }^{1} \mathrm{H}\right)=100 \mathrm{kHz}$. RD for ${ }^{1} \mathrm{H}$ NMR experiments was set to $5 \mathrm{~s}$. The chemical shift reference (0 ppm) for ${ }^{1} \mathrm{H}$ and ${ }^{31} \mathrm{P}$ was tetramethylsilane (TMS) $\mathrm{H}_{3} \mathrm{PO}_{4} 85 \%$, respectively.

\section{Results and discussion}

\subsection{Textural characterization}

\subsubsection{Nitrogen adsoption/desorption}

Nitrogen adsorption/desorption isotherms of SBA, SBA-ФP, SBA-NH and SBA-NH-ФP are of type IV according to the IUPAC classification, with a $\mathrm{H} 1$ type hysteresis loop characteristic of mesoporous solids (Fig. 1a). A surface area (SBET) of 766 and $355 \mathrm{~m}^{2} \mathrm{~g}^{-1}$ for the free-template SBA-15 (SBA) sample and for the amine-functionalized sample (SBA-NH), respectively, were calculated using the BET model [16]. Moreover, a narrow distribution of uniform pores is observed with an average pore diameter $\left(D_{\mathrm{P}}\right)$ of 60 and $54 \AA$ for SBA and SBA-NH, respectively that were determined with the BJH (Barrett-Joyner-Halenda) method based on the desorption branch of the isotherms [18] (Fig. 1b). Once loaded with phenylphosphonic acid a substantial reduction of the $S_{\mathrm{BET}}$ is found for both samples. We measured a specific surface area of 425 and $250 \mathrm{~m}^{2} \mathrm{~g}^{-1}$ for SBA- $\mathrm{SP}$ and SBA-NH- $\mathrm{SP}$, respectively. These values correspond to a decrease of the $\mathrm{S}_{\mathrm{BET}}$ of $45 \%$ and $30 \%$, respectively, when compared to the initial values of unloaded samples. The $D_{\mathrm{P}}$ are also decreasing down to 57 and $49 \AA$ for SBA-ФP and SBA-NH-ФP, respectively (Fig. 1b). These data suggests an 
Table 1

Textural characteristics of SBA-15-based materials before and after amine functionalization and loading with phenylphosphonic acid.

\begin{tabular}{|c|c|c|c|c|c|}
\hline & $\mathrm{S}_{\mathrm{BET}}\left(\mathrm{m}^{2} / \mathrm{g}\right)$ & $V_{\mathrm{P}}\left(\mathrm{cm}^{3} / \mathrm{g}\right)$ & $D_{\mathrm{P}}(\AA)$ & Isotherm type & Amount of phosphonic acid $(\mathrm{g} / \mathrm{g})$ \\
\hline SBA & 766 & 0.48 & 60 & IV & \\
\hline SBA-ФP & 425 & 0.43 & 57 & IV & 0.322 \\
\hline SBA-NH & 355 & 0.43 & 54 & IV & \\
\hline SBA-NH-ФP & 250 & 0.31 & 49 & IV & 0.380 \\
\hline
\end{tabular}

efficient encapsulation of $\Phi P$ molecules into the mesoporous framework. The textural characteristics are summarized in Table 1.

\subsubsection{Small angle XRD}

The small angle X-ray diffraction patterns of the SBA-15-based materials, before and after amine-functionalization and phenylphosphonic acid encapsulation are displayed in Supplementary Fig. S1. SBA sample is characterized by a single intense reflection peak (100) at $2 \theta$ angle around $1^{\circ}$ and two additional peaks at $1.7^{\circ}$ and $2^{\circ}$, due to the $(110)$ and $(200)$ reflections that are typical of a well ordered 2D hexagonal structure. After the introduction of amino groups (SBA-NH) and phosphonic acid loading (SBA-ФP and SBA-NH-ФP), the XRD patterns still exhibit strong (100) reflection peaks indicating that the $2 \mathrm{D}$ hexagonal mesoscopic order is preserved.

\subsubsection{TEM}

These conclusions were further supported by TEM observations (Fig. S2). The TEM images of the SBA-15-based materials are compared in Fig. S2. Each sample exhibits a 2D hexagonal mesostructure also after amine-functionalization and phosphonic acid loading.

\subsection{Efficiency of $\Phi P$ loading}

\subsubsection{Elemental analysis}

The amount of phenyl phosphonic acid molecules loaded in SBAsample was deduced from elemental analysis and was carefully calculated by taking into amount the amount of residual organic species (surfactant and ethoxy groups from TEOS). In the same manner, the amount of ФP loaded in the SBA-NH sample was calculated by taking into account the amount of aminopropyl groups. Hence, a quantity of 322 and $380 \mathrm{mg}$ of $\Phi \mathrm{P}$ per gram of porous materials for SBA- $\Phi$ P and SBA-NH- $\Phi P$, respectively, was determined. The higher amount found for SBA-NH-ФP could be explained by a greater affinity of the $\Phi P$ molecules for the aminopropyl groups rather than for the silanol groups present at the surface of SBA. This deduction is supported by the fact that the loading amount determined at each stage of the impregnation procedure is systematically higher (30\% to $50 \%$ more before the washing step) for SBA-NH-ФP than for SBA-ФP. (see Table S1).

\subsubsection{Wide angle $X R D$}

The wide-angle XRD analysis (Fig. 2) demonstrates that the $\Phi P$ phase is amorphous when trapped in SBA- $\Phi$ P and SBA-NH-ФP as no X-ray diffraction peaks are detected, whereas pure ФP is a crystalline solid at room temperature (RT). It indicates also that the washing step was efficient to remove the excess of $\Phi P$ molecules that did not enter the porous network and that could recrystallize outside the pores.

\subsection{Solid state NMR}

The SBA-NH sample was characterized by ${ }^{29} \mathrm{Si}$ MAS NMR under quantitative conditions (Fig. S3). The spectrum displays resonances of the $Q_{4}, Q_{3}$ and $Q_{2}$ silicates units at $-110,-100$ and $-91 \mathrm{ppm}$, respectively. The resonances associated to the organosilane species are visible at -67 and $-58 \mathrm{ppm}$ and correspond to the $T_{3}$ and $T_{2}$ units respectively, evidencing the efficient grafting of the aminopropyl groups (see also ${ }^{13} \mathrm{C} C \mathrm{CP}$ MAS spectra on Fig. S4). The integration of $\mathrm{T}$ and $\mathrm{Q}$ signals yields a $\mathrm{T} / \mathrm{Q}$ ratio of 0.2 , which is coherent with the amount of silane grafted obtained through TGA measurements $(2.5 \mathrm{mmol} / \mathrm{g})$.

${ }^{31}$ P MAS NMR spectroscopy of the two loaded samples was used here to study the inclusion of $\Phi P$ in the mesoporous materials (Fig. 3A). The ${ }^{31} \mathrm{P}$ MAS spectra of SBA- $\Phi$ P and SBA-NH-ФP show a main resonance located at 19.1 and $13.8 \mathrm{ppm}$, respectively whereas the ${ }^{31}$ P MAS spectrum of the bulk phenyl phosphonic acid displays a single resonance at $21.0 \mathrm{ppm}$. We note that the ${ }^{31} \mathrm{P}$ resonance in the SBA-NH-ФP sample is highly shifted compared to SBA-ФP. It is well known that the condensation of a phosphate or phosphonate moiety leads to an up-field shift of the ${ }^{31} \mathrm{P}$ resonance [19]. Thus, the ФP-surface interaction seems to be stronger when aminopropyl groups are present at the $\mathrm{SiO}_{2}$ surface. This 
statement sounds correct since phosphonates are acidic and amino groups are basic. The line width of the ${ }^{31} \mathrm{P}$ resonance of SBA-NH$\Phi P$ is 10 times larger than the one of SBA-ФP (10.4 vs. $1.0 \mathrm{ppm})$. This can be attributed to a wide distribution of chemical shifts caused by the $\Phi \mathrm{P} /$ aminopropyl interactions that lead to a disordered interface with a wide distribution of chemical environments around the phosphonate groups. Finally, we note that a small peak at $9.4 \mathrm{ppm}$ (30\% of the main resonance) and a weak shoulder centered at $6.3 \mathrm{ppm}$ (15\% of the main resonance) are visible on the SBA-ФP and the SBA-NH-ФP spectra, respectively. These latter signals could correspond to two phosphonate moieties condensed with each other through a P-O-P bond. Nevertheless these two resonances correspond to species in relative close proximity and do not correspond to species in different phases as cross-peaks are identified in the ${ }^{31} \mathrm{P}$ EXSY experiments (Fig. S5). This experi-ment is based on ${ }^{31} \mathrm{P}$ magnetization exchange through ${ }^{31} \mathrm{P}$ spin dif-fusion and thus allows the investigation of ${ }^{31} \mathrm{P}-{ }^{31} \mathrm{P}$ proximities.

The ${ }^{1} \mathrm{H}$ MAS spectrum (Fig. 3B) of SBA-NH- PP displays a broad signal centered at $6.8 \mathrm{ppm}(\mathrm{LW}=6 \mathrm{ppm})$ dominated by the proton resonances of encapsulated $\Phi P$ and aminopropyl groups. Similarly, the ${ }^{1} \mathrm{H}$ MAS spectrum of bulk $\Phi P$ displays a broad signal ( $\mathrm{LW}=6$ $\mathrm{ppm}$ ) corresponding mainly to the proton resonances of the phenyl ring and the $\mathrm{POH}$ groups. These two broad spectra are characteristic of rigid compounds where the strong ${ }^{1} \mathrm{H}-{ }^{1} \mathrm{H}$ homo-nuclear dipolar coupling dominates all other interactions [20]. On the contrary, the ${ }^{1} \mathrm{H}$ MAS spectrum of SBA-ФP displays a sharp res-onance peak centered at $7 \mathrm{ppm}$ that corresponds to the aromatic protons of encapsulated $\Phi P$. Such narrow resonances are unusual for such solid state samples and are due to a dynamical average of the ${ }^{1} \mathrm{H}-{ }^{1} \mathrm{H}$ homonuclear dipolar interaction due to the relative mobility of $\Phi P$ molecules in SBA-ФP. This result reveals the pres-ence of a confinement effect that decreases the thermodynamical parameters for ФP confined in SBA-15. Thus, at room temperature the dynamics of the guest molecule increases compared to the corresponding bulk substance. On the contrary, the host-guest interaction is stronger than the confinement effect in the case of SBA-NH- $\Phi$ P and leads to a rigid system.

The ${ }^{1} \mathrm{H}^{31} \mathrm{P}$ cross polarization (CP) experiment allows the transfer of the ${ }^{1} \mathrm{H}$ magnetization to the ${ }^{31} \mathrm{P}$ nuclei via the through space heteronuclear dipolar ${ }^{1} \mathrm{H}-{ }^{31} \mathrm{P}$ coupling. Consequently, the intensity of the ${ }^{31} \mathrm{P}$ signal is directly depending on the strength of the ${ }^{1} \mathrm{H}-{ }^{31} \mathrm{P}$ coupling. The Fig. $3 \mathrm{C}$ displays the variation of the 31P intensity as a function of the contact time (CT) during a ${ }^{1} \mathrm{H}-{ }^{31} \mathrm{P}$ CP MAS experiment for the samples SBA-ФP and SBA-NH-ФP (only the intensities of the main resonances are plotted). The evolutions of the ${ }^{31} \mathrm{P}$ intensity are drastically different for the two loaded samples. The two curves are fitted with the classical I-S model through the following equation that expresses the variation of the signal intensity as a function of two time constants [21]:

$$
\begin{aligned}
M_{31 P}\left(t_{\mathrm{CP}}\right)= & \left(\gamma_{1 H} / \gamma_{31 P}\right) M_{0}\left(1-T_{\mathrm{PH}} / T_{1 \rho}\right)^{-1}\left[\exp \left(-t_{\mathrm{CP}} / T_{1 \rho}\right)\right. \\
& \left.-\exp \left(-t_{\mathrm{CP}} / T_{\mathrm{PH}}\right)\right]
\end{aligned}
$$

where $t_{\mathrm{CP}}$ corresponds to the contact time, $M_{0}$ corresponds to the ${ }^{31} \mathrm{P}$ Zeeman magnetization, $T_{\mathrm{PH}}$ stands for the cross relaxation time constant and $T_{1 \rho}$ is the relaxation time of protons in the rotating frame. We found $T_{\mathrm{PH}}=0.66$ and $2.22 \mathrm{~ms}$, for SBA-NH-ФP and SBA- $\Phi P$ respectively. The first value is characteristic of a fast $C P$ transfer typical of a rigid system. The slowest behavior found for SBA-ФP can be explained by a higher mobility of ФP in SBA-ФP. The relatively long ${ }^{1} \mathrm{H}-{ }^{31} \mathrm{P}$ cross relaxation time $T_{\mathrm{PH}}$ constant for SBA- $\Phi \mathrm{P}$ can only be explained by a higher mobility of the $Ф P$ molecules at room temperature that averages out the ${ }^{1} \mathrm{H}-{ }^{31} \mathrm{P}$ heteronuclear dipolar interaction. The same behavior explains the narrow lines observed in the ${ }^{1} \mathrm{H}$ NMR spectrum of SBA-ФP. Furthermore, we note that the proton relaxation time in the rotating frame is also very different for the two samples. We found $T_{1 \rho}\left({ }^{1} \mathrm{H}\right)=1.79 \mathrm{~ms}$ for SBA-NH- $\mathrm{SP}$ whereas no $T_{1 \rho}\left({ }^{1} \mathrm{H}\right)$ effect was detected in the range $0-10 \mathrm{~ms}$ for SBA-ФP $\left(T_{1 \rho}\left({ }^{1} \mathrm{H}\right)>>10 \mathrm{~ms}\right)$. The longer $T_{1 \rho}\left({ }^{1} \mathrm{H}\right)$ found for SBA-ФP is also a sign of a higher mobility of $\Phi$ P molecules in SBA-ФP compared to SBA-NH-ФP [22]. In a general manner the dynamical properties of $\Phi P$ inside the mesoporous matrix leads to the average of the anisotropic interactions. A nice example concerns the chemical shift anisotropy (CSA) as shown in Fig. 3D that displays the ${ }^{31} \mathrm{P}$ static spectra of ФP and SBA-ФP. If bulk ФP exhibits a broad resonance corresponding to a CSA tensor (with the following shielding tensor components: $\delta_{11}=71 \mathrm{ppm} ; \delta_{22}=25 \mathrm{ppm} ; \delta_{33}=-29 \mathrm{ppm}$ ), SBA-ФP displays only one sharp resonance in which the CSA is average to the isotropic chemical shift. Interestingly, these data demonstrate that the dynamics is not concerning solely the phenyl ring but concerns the reorientation of the whole molecule.

As a partial conclusion, this higher mobility of ФP encapsulated in SBA-NH compared to bulk ФP is due to the so-called confinement effect that acts on small molecules confined in mesopores [10]. Similar effects were observed for carboxylic acids (benzoic and lauric acid) [23,24] and ibuprofen [9] encapsulated in MCM41 , where the liquid-solid phase transitions of such molecules were deeply depressed compared to the bulk and where they behave as liquid at room temperature.

\subsection{High magnetic field and fast MAS NMR experiments}

In solid state NMR, ${ }^{1} \mathrm{H}$ observation of rigid solids gives rise to spectra of poor resolution as it is observed for SBA-NH-ФP (Fig $3 \mathrm{~B})$ where the $1 \mathrm{H}$ resonances of the two organic components (the phenyl and the aminopropyl groups) are not discernable. This is mainly due to the presence of a strong ${ }^{1} \mathrm{H}-{ }^{1} \mathrm{H}$ homonuclear dipolar coupling that broadens the proton line shapes [20]. In order to average out this interaction and to increase the spectral resolution a possibility is to increase the static magnetic field and to increase the MAS speed rate [25]. This effect is illustrated in Fig. 4A. that displays various $1 \mathrm{H}$ MAS spectra of SBA-NH-ФP. The gain in resolution is spectacular passing from a static magnetic field $B_{0}$ of $7.0 \mathrm{~T}$ to $16.4 \mathrm{~T}$ and passing from moderate MAS spin rate $(14 \mathrm{kHz})$ to high spinning rate. The increase of the spinning speed $v_{\text {MAS }}$ from 40 to $67 \mathrm{kHz}$ enhances also significantly the spectral resolution. At this stage the ${ }^{1} \mathrm{H}$ resonances of the two organic components are resolved: the phenyl group from $Ф P$ is signing around $7.0 \mathrm{ppm}$ whereas the aminopropyl resonances are found between 0 and $3 \mathrm{ppm}$. The complete assignment of the proton signals can be realized through a $2 \mathrm{D}{ }^{1} \mathrm{H}-{ }^{13} \mathrm{C}$ HetCor experiment (Fig. S6) that allows the correlation of ${ }^{1} \mathrm{H}$ and ${ }^{13} \mathrm{C}$ spins that are spatially coupled (through space correlation via ${ }^{1} \mathrm{H}-{ }^{13} \mathrm{C}$ dipolar coupling). Each ${ }^{13} \mathrm{C}$ 
resonance of the amnipropyl group is correlating with one single shoulder of the ${ }^{1} \mathrm{H}$ corresponding signal at $\delta=0.7 \mathrm{ppm}\left(\mathrm{NH}_{2}-\right.$ $\left.\mathrm{CH}_{2}-\mathrm{CH}_{2}-\mathbf{C H}_{2}-\mathrm{Si}\right), \quad \delta=1.7 \mathrm{ppm}\left(\mathrm{NH}_{2}-\mathrm{CH}_{2}-\mathbf{C H}_{2}-\mathrm{CH}_{2}-\mathrm{Si}\right)$ and $\delta=2.7 \mathrm{ppm}\left(\mathrm{NH}_{2}-\mathrm{CH}_{2}-\mathrm{CH}_{2}-\mathrm{CH}_{2}-\mathrm{Si}\right)$.

The high resolution ${ }^{1} \mathrm{H}$ spectra $\left(B_{0}=16.4 \mathrm{~T}, v_{\mathrm{MAS}}=67 \mathrm{kHz}\right)$ of SBA-NH, ФP, and SBA- $\Phi$ P are displayed on Fig. 4B. The $1 \mathrm{H}$ spectrum of SBA-NH display the three characteristic resonances of the aminopropyl groups described above though they are narrower probably due to a greater mobility in the absence of $\Phi P$. The ${ }^{1} \mathrm{H}$ spectrum of SBA-ФP displays the characteristic resonances of the proton phenyl ring centered at $7.0 \mathrm{ppm}$. Nevertheless, one can note that no gain in spectral resolution is observed passing from moderate to high $B_{0}$ and $v_{\text {MAs. }}$. The similar ${ }^{1} \mathrm{H}$ line widths ( $\left.\sim 1 \mathrm{ppm}\right)$ observed in Fig. 3B $(B 0=7.0 \mathrm{~T}, v M A S=14 \mathrm{kHz})$ and in Fig. $4 \mathrm{~B}$ $\left(\mathrm{B} 0=16.4 \mathrm{~T}, v_{\mathrm{MAS}}=67 \mathrm{kHz}\right)$.

In the case of the $\varphi \mathrm{P}$, the ${ }^{1} \mathrm{H}$ spectrum exhibits the resonances of the phenyl ring at 6.6 and 8 ppm together with a deshielded resonance at $12 \mathrm{ppm}$ corresponding to the $\mathrm{POH}$ groups of the phosphonate (for a complete assignment see [26]). Interestingly, the resonance of hydrogen phosphate moiety is absent on the ${ }^{1} \mathrm{H}$ spectra of SBA-ФP and SBA-NH-ФP. Two reasons can explain such an observation. (i) $\varphi \mathrm{P}$ molecules are deprotonated inside the material. This assumption is credible in the case of SBA-NH-ФP where the " $\varphi \mathrm{P} / \mathrm{aminopropyl"} \mathrm{interface} \mathrm{can} \mathrm{be} \mathrm{governed} \mathrm{by} \mathrm{an} \mathrm{acid-base} \mathrm{like}$ interaction in reason of their respective $\mathrm{pKa}$ ( $\mathrm{pKa} \sim 9$ and 2 for the amine and the phosphonate moiety, respectively). Then, a strong ionic-like interaction can be supposed as follow: $\mathrm{NH}_{3}{ }^{+} \ldots-$ OP. (ii) The $\mathrm{H}^{+}$of the $\mathrm{POH}$ is involved in a chemical exchange and is not detected at room temperature (RT) by NMR. This assumption is possible for SBA-ФP where the acidic POH groups can exchange with residual $\mathrm{H}_{2} \mathrm{O}$ and/or silanols $\mathrm{SiOH}$ present at the surface of the material. This phenomenon has been evidenced for various carboxylic acids encapsulated in mesoporous silica and in particular for ibuprofen trapped in MCM-41 [9]. Similarly, the $\mathrm{H}^{+}$ from the carboxyl group $\mathrm{COOH}$ is engaged in a fast chemical exchange at RT and thus is not detected by ${ }^{1} \mathrm{H}$ NMR. Only low temperature ${ }^{1} \mathrm{H}$ MAS NMR experiments (usually down to $170 \mathrm{~K}$ ) could evidence this phenomenon.

Until now, one ${ }^{1} \mathrm{H}$ signal has not been discussed and concerns the amino protons. Proton proximity can be studied through $2 \mathrm{D}$ double quantum (DQ) ${ }^{1} \mathrm{H}-{ }^{1} \mathrm{H}$ experiments based on ${ }^{1} \mathrm{H}-{ }^{1} \mathrm{H}$ homonuclear dipolar coupling [27]. Fig. 5 displays such an experiment for SBA-NH. The on-diagonal signals (that correspond to autocorrelations peaks) are observed for each aminopropyl ${ }^{1} \mathrm{H}$ resonances and are characteristic of the density of these species inside the material. Moreover, a signal of lower intensity at $7.5 \mathrm{ppm}$ is remarkable because it exhibits an off-diagonal correlation peak with only one propyl signal at $2.7 \mathrm{ppm}\left(\mathrm{NH}_{2}-\mathbf{C H}_{2}-\mathrm{CH}_{2}-\mathrm{CH}_{2}-\mathrm{Si}\right)$. Thus, the signal at $7.5 \mathrm{ppm}$ can be assigned to the amine protons $\mathrm{NH}$. Furthermore, one can note that the signal at $6 \mathrm{ppm}$ visible on the ${ }^{1} \mathrm{H}$ MAS spectrum is not detected in the DQ spectrum. We assign this resonance to adsorbed water for which the ${ }^{1} \mathrm{H}-{ }^{1} \mathrm{H}$ homonuclear dipolar coupling is average out due its dynamical properties.
We use a similar experiment in order to explore the host/guest interface in SBA-NH-ФP (Fig. 6). The autocorrelation peaks of the propyl groups are visible as well as those of the phenyl protons. Moreover, intense off-diagonal peaks are revealed. They correspond to correlations between phenyl groups of encapsulated $\varphi P$ and aminopropyl groups grafted onto the silica pore walls. This result highlights that a strong interaction exists in the materials between the matrix and the guest molecules associated to a short a ${ }^{1} \mathrm{H}-{ }^{1} \mathrm{H}$ distance $(\mathrm{d}<5-6 \AA$ ) [20]. Furthermore, a careful analysis of the $2 \mathrm{D}$ spectrum reveals that the phenyl display a correlation peak with each ${ }^{1} \mathrm{H}$ from the propyl group that implies a particular conformation of $\varphi \mathrm{P}$ regarding the aminopropyl moiety. The Fig. 7 displays two conformations of $\varphi P$ in the pore matrix. In the two schematic representations the angle $\mathrm{NH}-\mathrm{O}$ was fixed at $180^{\circ}$ and the distance $\mathrm{NH}$... O was fixed at $1.1 \AA$ assuming a strong $\mathrm{H}$-bond between the amine and the phosphonate groups. If the phosphonate is standing along the axis of the aminopropyl chain (i.e. if the phenyl group is pointing to the center of the pore) the distance between protons from the phenyl ring and the $\mathrm{CH}_{2}$ bonded to $\mathrm{Si}$ is too long to give rise to a $2 \mathrm{Q}$ correlation peak (Fig. $7 \mathrm{~A}$ ). Indeed, the distance relative to the $\mathrm{H}$ in ortho position is $8.4 \AA$. Of course, distances relative to $\mathrm{H}$ in meta and para position are even longer. Fig. 7B displays a more probable conformation with the phenyl ring pointing to the surface of the silica wall. In this case, distances between hydrogen from the phenyl ring and the aminopropyl group are standing between 5.0 and $5.7 \AA$ which are compatible with the cross peaks observed on $2 \mathrm{D} D Q{ }^{1} \mathrm{H}$ spectrum. Such a conformation could be explained by stabilization through weak bonds between the phenyl ring and the propyl chain (London dispersions forces).

\section{Conclusion}

We demonstrate in this communication that $\varphi \mathrm{P}$ can be efficiently loaded in mesoporous SBA-15 and aminopropyl-modified SBA powdered samples through the incipient wetness impregnation method. The phosphonic acid molecules that do not crystallize inside the pores, present two radically different behaviors: $(i)$ in SBA-15 the molecules are highly mobile and are submitted to a confinement effect due to the mesoscopic size of the pores and present a weak interaction molecule-silica. (ii) In aminopropylmodified SBA material, the molecules are rigid and are in strong interaction with the aminopropyl groups. 2D double quantum ${ }^{1} \mathrm{H}$ NMR experiments recorded at high field and high spinning speed permit to propose a model of the molecule-aminopropyl interaction. The increase in spectral resolution due to the combination of high magnetic field and fast MAS rate allows also the assignment of ${ }^{1} \mathrm{H}$ resonances in aminopropyl-modified SBA matrix and notably allows the assignment of the protons resonance of the amino group.

\section{Appendix A. Supplementary data}

Supplementary data associated with this article can be found, in the online version, at http://dx.doi.org/10.1016/j.micromeso.2012. 04.028 .

\section{References}

[1] M. Vallet-Regi, A. Ramila, R.P. del Real, J. Perez-Pariente, Chem. Mater. 13 (2001) 308.

[2] M. Vallet-Regi, F. Balas, D. Arcos, Angew. Chem. Int. Ed. 46 (2007) 7548

[3] a) P. Horcajada, A. Ramila, J. Perez-Pariente, M. Vallet-Regi, Micro. Meso. Mater. 68 (2004) 105;

b) M. Van Speybroeck, R. Mellaerts, R. Mols, T. Do Thi, J.A. Martens, J.V. Humbeeck, P. Annaert, G. Van den Mooter, P. Augustijns, Eur. J. Pharm. Sci. 41 (2010) 623

[4] J. Andersson, J. Rosenholm, S. Areva, M. Linden, Chem. Mater. 16 (2004) 4160. 
[5] L. Contessotto, E. Ghedini, F. Pinna, M. Signoretto, G. Cerrato, V. Crocella, Chem. A Eur. J. 15 (2009) 12043.

[6] B.G. Trewyn, I.I. Slowing, S. Giri, H.-T. Chen, V.S.-Y. Lin, Acc. Chem. Res. 40 (2007) 846.

[7] P.A. Tishmack, D.E. Bugay, S.R. Byrn, J. Pharm. Sci. 92 (2003) 441.

[8] C. Bonhomme, C. Coelho, N. Baccile, C. Gervais, T. Azaïs, F. Babonneau, Acc. Chem. Res. 40 (2007) 738.

[9] T. Azaïs, C. Tourné-Pethéil, F. Aussenac, N. Baccile, C. Coelho, J.-M. Devoisselle, F. Babonneau, Chem. Mater. 18 (2006) 6382.

[10] C. Alba-Simionesco, B. Coasne, G. Dosseh, G. Dudziak, K.E. Gubbins, R. Radhakrishnan, M. Sliwinska-Bartkowiak, J. Phys. Condens.: Matter. 18 (2006) R15.

[11] D. Zhao, J. Feng, Q. Huo, N. Melosh, G.H. Fredrickson, B.F. Chmelka, G.D. Stucky, Science 279 (1998) 548.

[12] F. Balas, M. Manzano, P. Horcajada, M. Vallet-Regi, J. Am. Chem. Soc. 128 (2006) 8116

[13] B. Munoz, A. Ramila, J. Perez-Pariente, I. Diaz, M. Vallet-Regi, Chem. Mater. 15 (2003) 500 .

[14] A. Nieto, M. Colilla, F. Balas, M. Vallet-Regi, Langmuir 26 (2010) 5038.

[15] C. Charnay, S. Begu, C. Tourne-Peteilh, L. Nicole, D.A. Lerner, J.-M. Devoisselle, Eur. Pharm. Biopharm. 57 (2004) 533.
[16] S. Brunauer, P.H. Emmett, E.J. Teller, J. Am. Chem. Soc. 62 (1938) 309.

[17] A. Galarneau, D. Desplantier, R. Dutartre, F. Di Renzo, Micro. Meso. Mater. 27 (1999) 297.

[18] P. Barrett, L.G. Joyner, P. Halenda, J. Am. Chem. Soc. 73 (1951) 373.

[19] M.M. Crutchfield, C.F. Callis, R.R. Irani, G.C. Roth, Inorg. Chem. 4 (1962) 813.

[20] S.P. Brown, Solid State Nucl. Magn. Reson. (2011), http://dx.doi.org/10.1016/ j.ssnmr.2011.11.006

[21] A. Pines, M. Gribby, J. Waugh, J. Chem. Phys. 59 (1973) 569.

[22] K. Schmidt-Rohr, H.W. Spiess, Acad. Press (1996).

[23] T. Azaïs, G. Hartmeyer, S. Quignard, G. Laurent, C. Tourné-Péteilh, J-M. Devoisselle, F. Babonneau, Pure Appl. Chem. 81 (2009) 1345.

[24] T. Azaïs, G. Hartmeyer, S. Quignard, G. Laurent, F. Babonneau, J. Phys. Chem. C $114(2010) 8884$.

[25] T. Azaïs, L. Bonhomme-Coury, J. Vaissermann, J. Maquet, C. Bonhomme, Eur. J. Inorg. Chem. (2002) 2838

[26] C. Gervais, M. Profeta, V. Lafond, C. Bonhomme, T Azaïs, H. Mutin, C.J. Pickard, F. Mauri, F. Babonneau, Magn. Reson. Chem. 42 (2004) 445.

[27] M. Feike, D.E. Demco, R. Graf, J. Gottwald, S. Hafner, H.W. Spiess, Ser. A 122 (1996) 214 

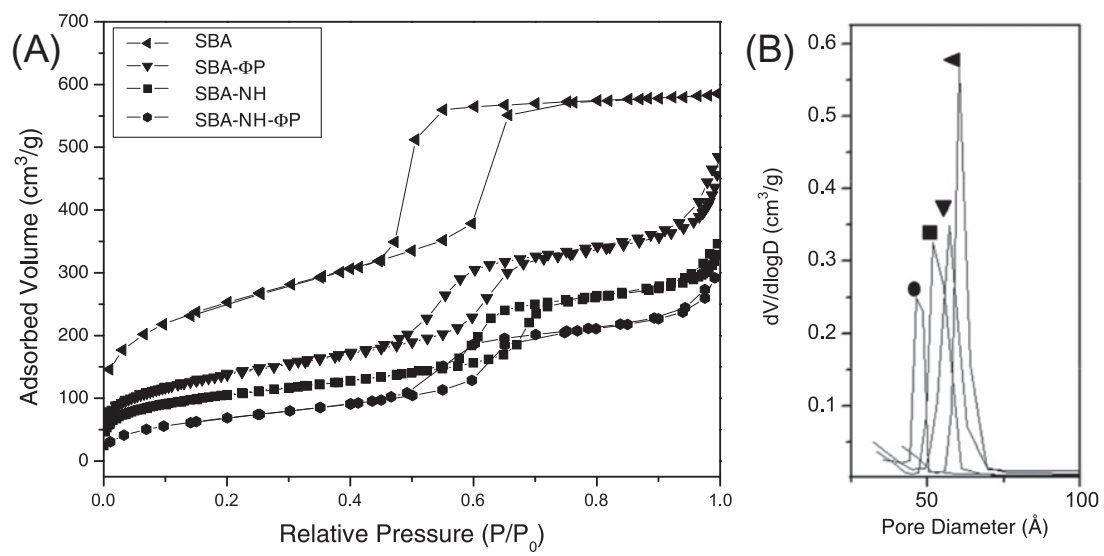

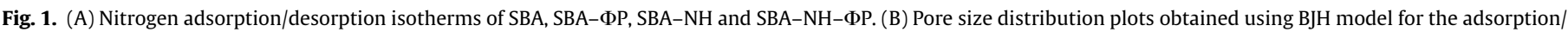
desorption branch isotherm of SBA, SBA-ФP, SBA-NH and SBA-NH-ФP.

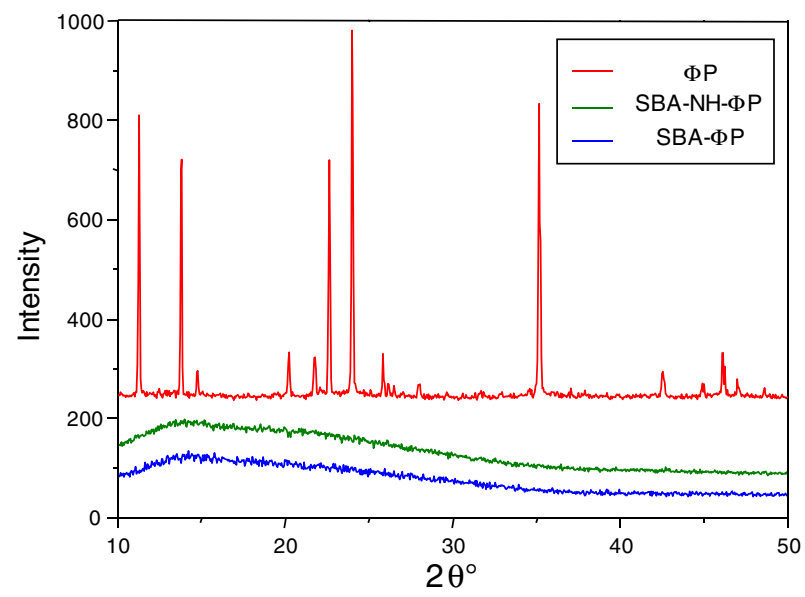

Fig. 2. Wide angle XRD pattern of SBA-ФP, SBA-NH-ФP and $\Phi$ P. 

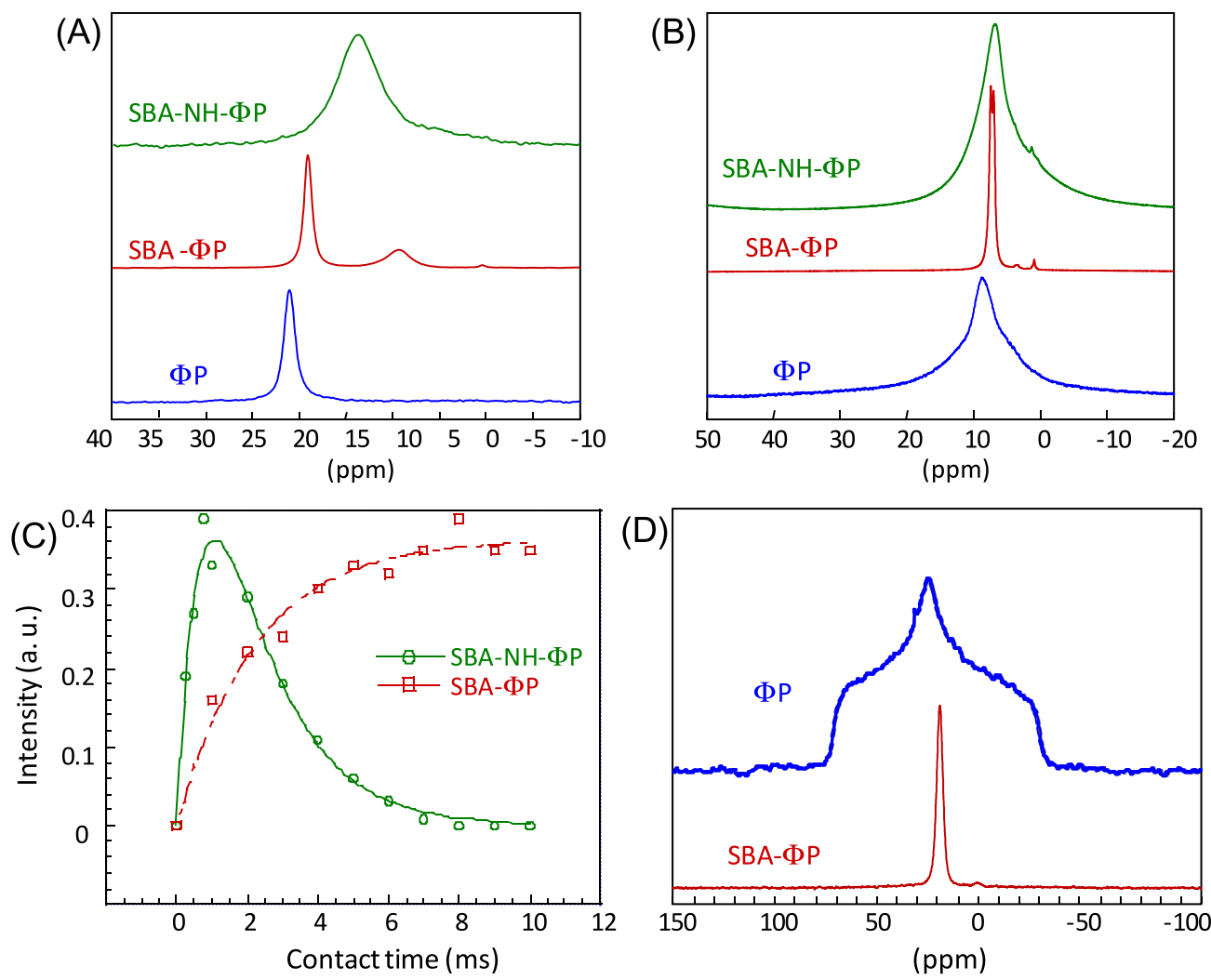

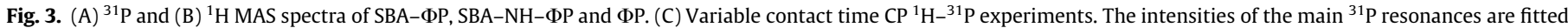
using Eq. (1) (see Section 3). (D) Static ${ }^{31} \mathrm{P}$ spectra of SBA-ФP, and ФP recorded at RT. 

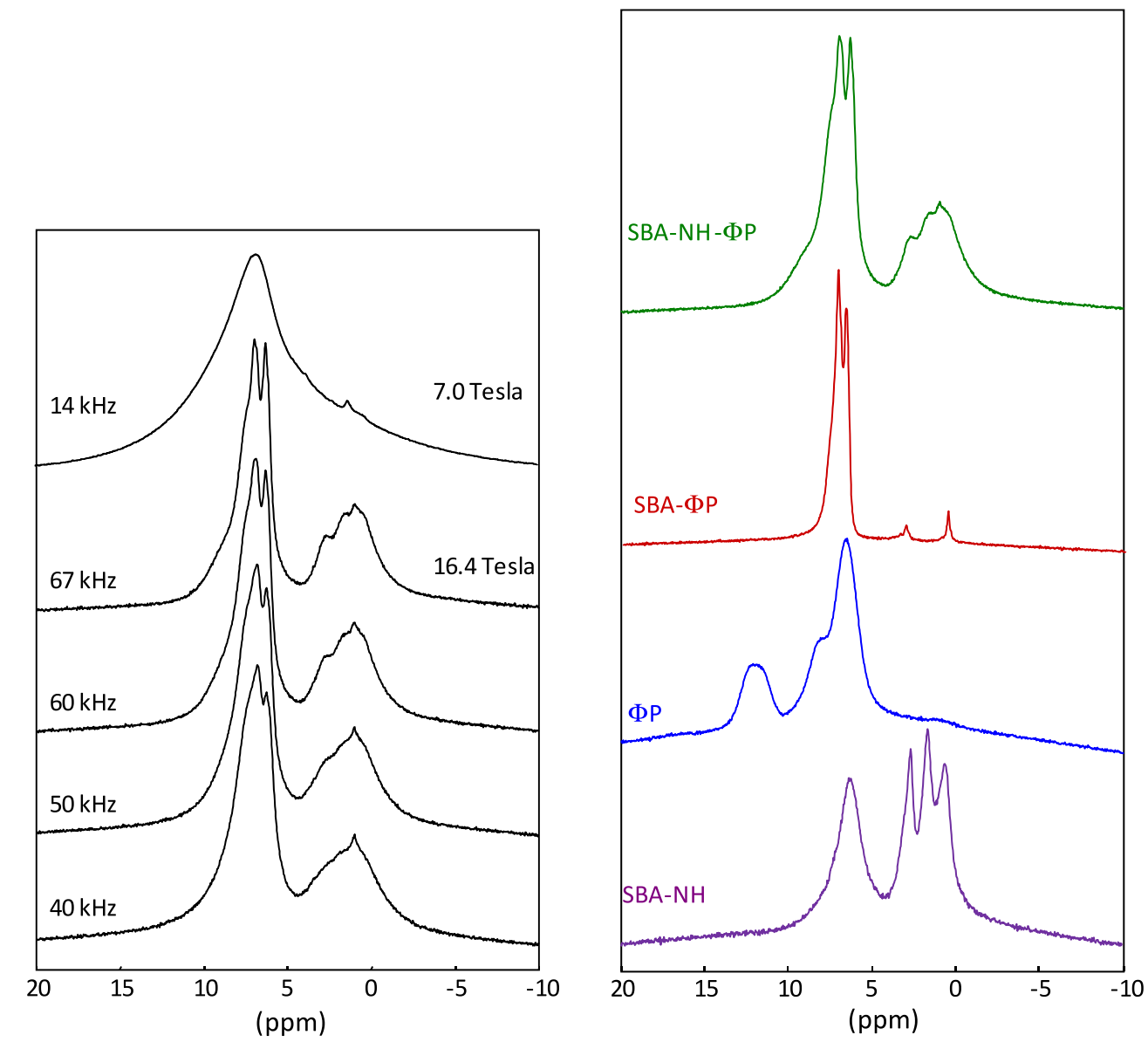

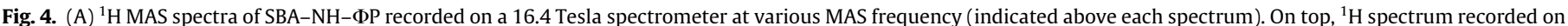

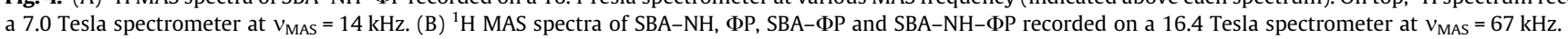




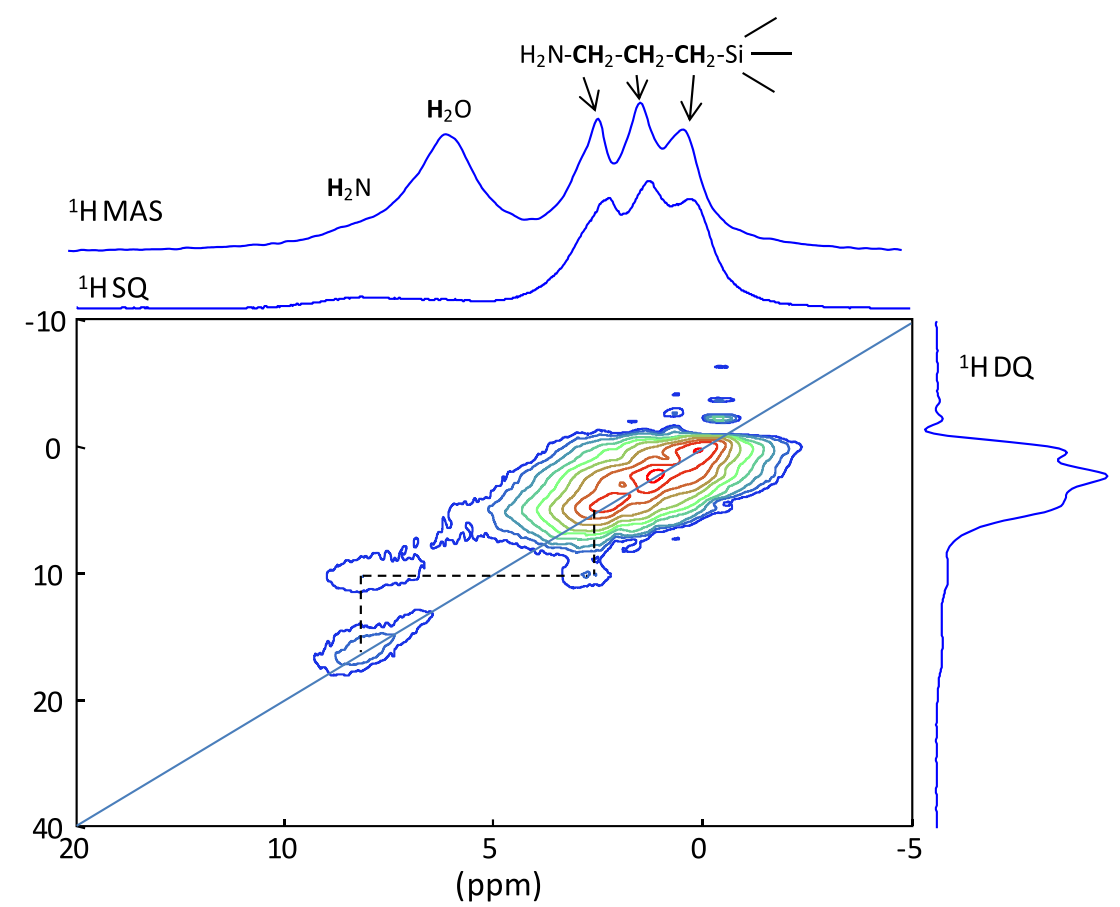

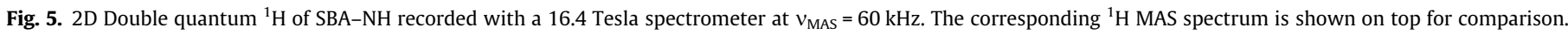

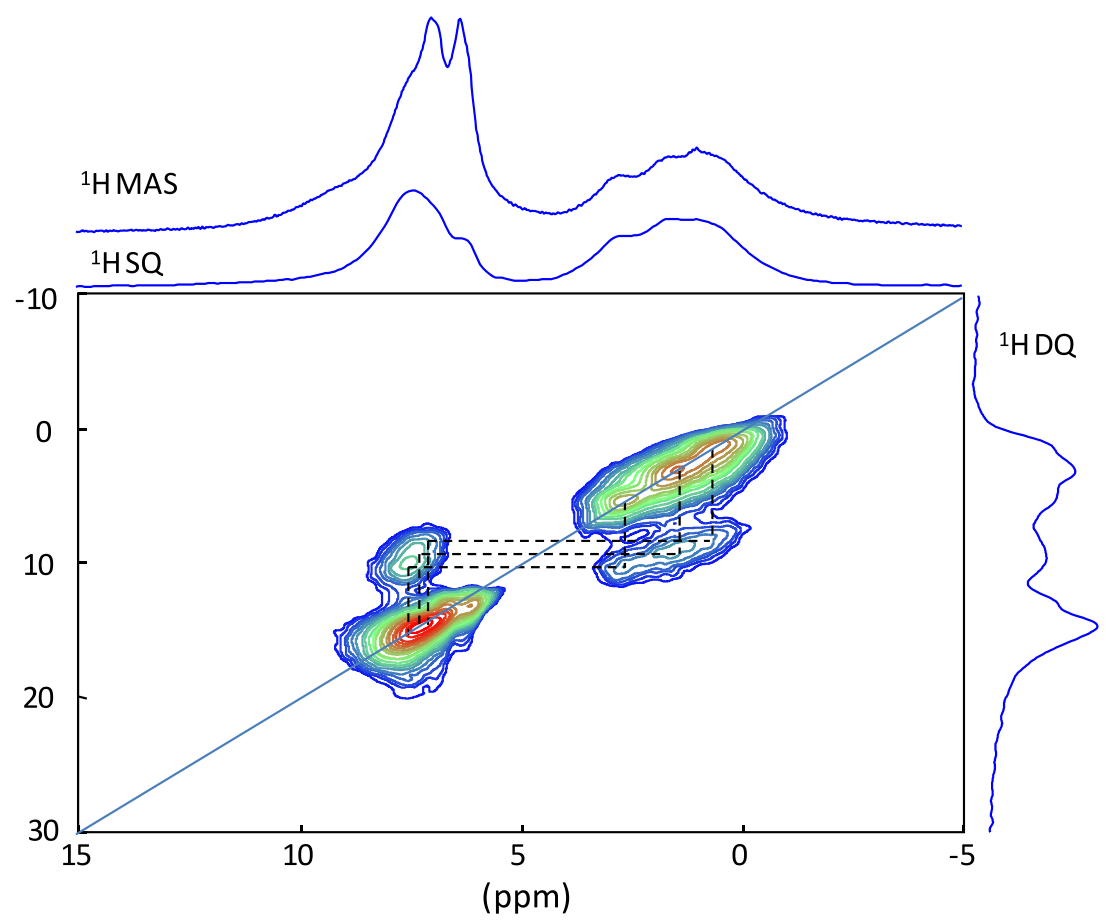

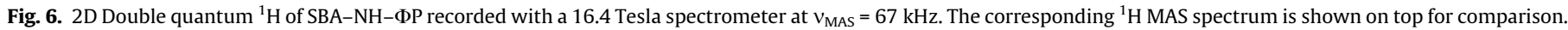




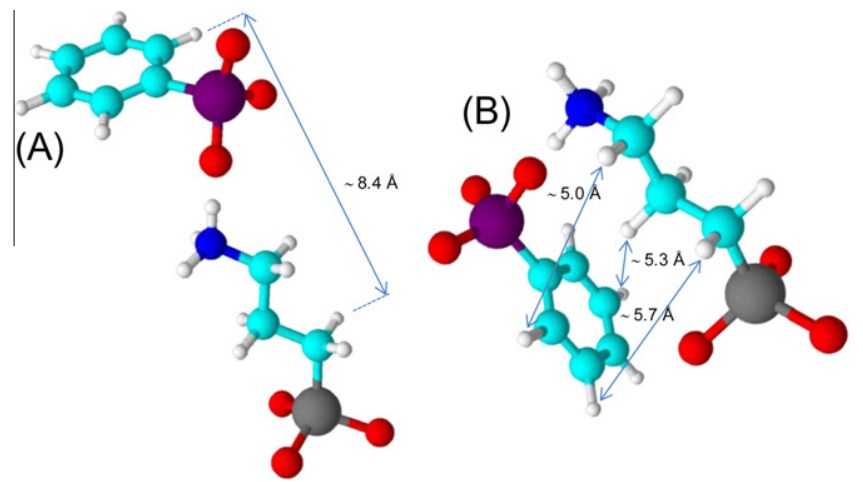

Fig. 7. Schematic representation of two possible "ФP - aminopropyl" conformations in SBA-NH-ФP with selected ${ }^{1} \mathrm{H}-{ }^{1} \mathrm{H}$ distances. In the two representation, the angle $\mathrm{NH}-\mathrm{O}$ was fixed at $180^{\circ}$ and the distance $\mathrm{NH} \cdots \mathrm{O}$ was fixed at $1.1 \AA$ assuming a strong $\mathrm{H}$-bond between the amine and the phosphonate groups ( $\mathrm{Si}$ : gray; $\mathrm{P}$ : purple; N: dark blue; C: light blue; O: red; H:white) (For interpretation of the references to color in this figure legend, the reader is referred to the web version of this article.). 